\title{
ON IMPULSIVE SEMIDYNAMICAL SYSTEMS: MINIMAL, RECURRENT AND ALMOST PERIODIC MOTIONS
}

\author{
Everaldo M. Bonotto - Manuel Z. Jimenez
}

\begin{abstract}
This paper concerns results about minimal, recurrent and almost periodic motions in impulsive semidynamical systems. In the first part, we investigate general properties of minimal sets. In the sequel, we study some relations among minimal, recurrent and almost periodic motions. Some important results from the classical dynamical systems theory are generalized to the impulsive case, as Birkhoff's theorem for instance.
\end{abstract}

\section{Introduction}

Impulsive differential equations describe the evolution of systems where the continuous development of a process is interrupted by abrupt changes of state. The reader may find some important results and applications of impulsive systems in the papers [1]-[3], [12], [16]-[18], for instance.

In the last years, the action of impulses on impulsive semidynamical systems has been intensively investigated. The study of this theory started by V. Rozko on the paper [22] published in 1972. In [22] and [23], Rozko studied a class of almost periodic motions in pulsed system and the theory of stability in terms of Lyapunov for impulsive systems. Later on, Saroop Kaul continued the study in impulsive systems presenting several important results as recursiveness and theory of stability, see [19]-[21]. In 2004 several important results due Ciesielski

2010 Mathematics Subject Classification. Primary 37B25; Secondary 34A37, 54H20.

Key words and phrases. Semidynamical systems, minimal sets, recurrence, impulses.

The first named authoor supportedby FAPESP 2010/08994-7.

The second named author supported by FAPESP 2010/12250-3. 
were published in this area. In [13] and [14], Ciesielski proves results which concern the continuity of the function which describes the time of reaching impulse points, invariancy and stability. In [15] is presented theorems of isomorphisms of impulsive systems. Results which deal with asymptotic stability, flows of characteristic $0^{+}$, theorem of Poincaré-Bendixson, Poisson stability, LaSalle's principle, functional of Lyapunov and dissipative systems may be found in [5]-[11].

However, many results from the classical continuous dynamical systems theory have not been studied for discontinuous systems. In particular, results which deal with minimal, recurrent and almost periodic motions still need to be investigated when the system is perturbed by abrupt changes. The aim of this paper is to establish general properties of minimal sets and show results which relate minimal, recurrent and almost periodic motions. In the next lines we describe the organization of the paper and the main results.

We start by presenting a summary of the basis of semidynamical systems with impulse effects. In Section 2, we give some basic definitions and notations about impulsive semidynamical systems. In Section 3, we present some additional definitions and results that will be very useful in this paper. Section 4, concerns the main results of this paper. In the sequel, we mention some of these results.

It is well know that every nonempty compact invariant set contains a compact minimal set, see Theorem 4.4 (Chapter III) in [4] for instance. We provide sufficient conditions to obtain a similar result for impulsive semidynamical systems. The result is presented in Theorem 4.13 .

The version of Birkhoff's theorem is presented in Theorem 4.17. Given an impulsive system, we show that every point of a minimal set that is not an impulsive point is recurrent. Also, we show that in complete metric spaces the closure of any recurrent trajectory is compact and minimal under some conditions, see Theorem 4.18 .

For continuous dynamical systems, it is known that an almost periodic point which possesses the closure of its trajectory compact is a recurrent point. We also have this result for impulsive systems and it is presented in Theorem 4.23. In [4], for the continuous case, the authors use the uniform continuity of the trajectories to obtain the proof. Since we do not have uniform continuity for impulsive systems we point out another type of proof that can be used for continuous systems.

In Theorem 4.24, we consider a complete metric space and we suppose that the positive orbit of a point together with its impulsive points form a minimal set. Then we present conditions for this point to be eventually periodic. And in Theorem 4.25, we establish conditions for an eventually periodic point to be periodic. 


\section{Preliminaries}

Let $X$ be a metric space and $\mathbb{R}_{+}$be the set of non-negative real numbers. The triple $\left(X, \pi, \mathbb{R}_{+}\right)$is called a semidynamical system, if the function $\pi: X \times \mathbb{R}_{+} \rightarrow X$ is continuous with $\pi(x, 0)=x$ and $\pi(\pi(x, t), s)=\pi(x, t+s)$, for all $x \in X$ and $t, s \in \mathbb{R}_{+}$. We denote such system simply by $(X, \pi)$. For every $x \in X$, we consider the continuous function $\pi_{x}: \mathbb{R}_{+} \rightarrow X$ given by $\pi_{x}(t)=\pi(x, t)$ and we call it the motion of $x$.

Let $(X, \pi)$ be a semidynamical system. Given $x \in X$, the positive orbit of $x$ is given by $\pi^{+}(x)=\left\{\pi(x, t): t \in \mathbb{R}_{+}\right\}$. For $t \geq 0$ and $x \in X$, we define $F(x, t)=\{y \in X: \pi(y, t)=x\}$ and, for $\Delta \subset[0,+\infty)$ and $D \subset X$, we define

$$
F(D, \Delta)=\bigcup\{F(x, t): x \in D \text { and } t \in \Delta\} .
$$

Then a point $x \in X$ is called an initial point if $F(x, t)=\emptyset$ for all $t>0$.

An impulsive semidynamical system $(X, \pi ; M, I)$ consists of a semidynamical system, $(X, \pi)$, a nonempty closed subset $M$ of $X$ such that for every $x \in M$, there exists $\varepsilon_{x}>0$ such that

$$
F\left(x,\left(0, \varepsilon_{x}\right)\right) \cap M=\emptyset \quad \text { and } \quad \pi\left(x,\left(0, \varepsilon_{x}\right)\right) \cap M=\emptyset,
$$

and a continuous function $I: M \rightarrow X$ whose action we explain below in the description of the impulsive trajectory of an impulsive semidynamical system. The set $M$ is called the impulsive set and the function $I$ is called impulse function. We also define

$$
M^{+}(x)=\left(\bigcup_{t>0} \pi(x, t)\right) \cap M .
$$

Let $(X, \pi ; M, I)$ be an impulsive semidynamical system. We define the function $\phi: X \rightarrow(0,+\infty]$ by

$$
\phi(x)= \begin{cases}s & \text { if } \pi(x, s) \in M \text { and } \pi(x, t) \notin M \text { for } 0<t<s, \\ +\infty & \text { if } M^{+}(x)=\emptyset .\end{cases}
$$

This means that $\phi(x)$ is the least positive time for which the trajectory of $x$ meets $M$. Thus for each $x \in X$, we call $\pi(x, \phi(x))$ the impulsive point of $x$.

The impulsive trajectory of $x$ in $(X, \pi ; M, I)$ is an $X$-valued function $\widetilde{\pi}_{x}$ defined on the subset $[0, s)$ of $\mathbb{R}_{+}(s$ may be $+\infty)$. The description of such trajectory follows inductively as described in the following lines.

If $M^{+}(x)=\emptyset$, then $\widetilde{\pi}_{x}(t)=\pi(x, t)$ for all $t \in \mathbb{R}_{+}$and $\phi(x)=+\infty$. However, if $M^{+}(x) \neq \emptyset$, there is the smallest positive number $s_{0}$ such that $\pi\left(x, s_{0}\right)=x_{1} \in$ $M$ and $\pi(x, t) \notin M$, for $0<t<s_{0}$. Then we define $\widetilde{\pi}_{x}$ on $\left[0, s_{0}\right]$ by

$$
\tilde{\pi}_{x}(t)= \begin{cases}\pi(x, t), & 0 \leq t<s_{0}, \\ x_{1}^{+}, & t=s_{0},\end{cases}
$$


where $x_{1}^{+}=I\left(x_{1}\right)$ and $\phi(x)=s_{0}$. Let us denote $x$ by $x_{0}^{+}$.

Since $s_{0}<+\infty$, the process now continues from $x_{1}^{+}$onwards. If $M^{+}\left(x_{1}^{+}\right)=\emptyset$, then we define $\widetilde{\pi}_{x}(t)=\pi\left(x_{1}^{+}, t-s_{0}\right)$ for $s_{0} \leq t<+\infty$ and $\phi\left(x_{1}^{+}\right)=+\infty$. When $M^{+}\left(x_{1}^{+}\right) \neq \emptyset$, there is the smallest positive number $s_{1}$ such that $\pi\left(x_{1}^{+}, s_{1}\right)=$ $x_{2} \in M$ and $\pi\left(x_{1}^{+}, t-s_{0}\right) \notin M$, for $s_{0}<t<s_{0}+s_{1}$. Then we define $\widetilde{\pi}_{x}$ on $\left[s_{0}, s_{0}+s_{1}\right]$ by

$$
\tilde{\pi}_{x}(t)= \begin{cases}\pi\left(x_{1}^{+}, t-s_{0}\right), & s_{0} \leq t<s_{0}+s_{1}, \\ x_{2}^{+}, & t=s_{0}+s_{1},\end{cases}
$$

where $x_{2}^{+}=I\left(x_{2}\right)$ and $\phi\left(x_{1}^{+}\right)=s_{1}$, and so on. Notice that $\widetilde{\pi}_{x}$ is defined on each interval $\left[t_{n}, t_{n+1}\right]$, where $t_{0}=0$ and $t_{n+1}=\sum_{i=0}^{n} s_{i}, n=0,1, \ldots$ Hence $\widetilde{\pi}_{x}$ is defined on $\left[0, t_{n+1}\right]$.

The process above ends after a finite number of steps, whenever $M^{+}\left(x_{n}^{+}\right)=\emptyset$ for some $n$. Or it continues infinitely, if $M^{+}\left(x_{n}^{+}\right) \neq \emptyset, n=0,1, \ldots$, and in this case the function $\widetilde{\pi}_{x}$ is defined on the interval $[0, T(x))$, where $T(x)=\sum_{i=0}^{\infty} s_{i}$.

Let $(X, \pi ; M, I)$ be an impulsive semidynamical system. Given $x \in X$, the impulsive positive orbit of $x$ is defined by the set

$$
\widetilde{\pi}^{+}(x)=\{\widetilde{\pi}(x, t): t \in[0, T(x))\} .
$$

Analogously to the non-impulsive case, an impulsive semidynamical system satisfies the following standard properties: $\widetilde{\pi}(x, 0)=x$ for all $x \in X$ and $\widetilde{\pi}(\widetilde{\pi}(x, t), s)=\widetilde{\pi}(x, t+s)$, for all $x \in X$ and for all $t, s \in[0, T(x))$ such that $t+s \in[0, T(x))$. See [5] for a proof of it.

For details about the structure of these types of impulsive semidynamical systems, the reader may consult [8], [5], [10] and [13], [14], [15], [19], [20].

\section{Additional definitions and results}

Let us consider a metric space $X$ with metric $d$. By $B(x, \delta)$ we mean the open ball with center at $x \in X$ and radius $\delta>0$. Given $A \subset X$, let $B(A, \delta)=$ $\{x \in X: d(x, A)<\delta\}$ where $d(x, A)=\inf \{d(x, y): y \in A\}$. By $A^{c}$ we denote the complement of the set $A$ in $X$.

Let $(X, \pi)$ be a semidynamical system. Any closed set $S \subset X$ containing $x$ $(x \in X)$ is called a section or a $\lambda$-section through $x$, with $\lambda>0$, if there exists a closed set $L \subset X$ such that

(a) $F(L, \lambda)=S$;

(b) $F(L,[0,2 \lambda])$ is a neighbourhood of $x$;

(c) $F(L, \mu) \cap F(L, \nu)=\emptyset$, for $0 \leq \mu<\nu \leq 2 \lambda$. 
The set $F(L,[0,2 \lambda])$ is called a tube or a $\lambda$-tube and the set $L$ is called a bar. Let $(X, \pi ; M, I)$ be an impulsive semidynamical system. We now present the conditions TC and STC for a tube.

Any tube $F(L,[0,2 \lambda])$ given by a section $S$ through $x \in X$ such that $S \subset$ $M \cap F(L,[0,2 \lambda])$ is called TC-tube on $x$. We say that a point $x \in M$ fulfills the Tube Condition and we write (TC), if there exists a TC-tube $F(L,[0,2 \lambda])$ through $x$. In particular, if $S=M \cap F(L,[0,2 \lambda])$ we have a STC-tube on $x$ and we say that a point $x \in M$ fulfills the Strong Tube Condition (we write (STC)), if there exists a STC-tube $F(L,[0,2 \lambda])$ through $x$.

The following theorem concerns the continuity of $\phi$ which is accomplished outside $M$ for $M$ satisfying the condition TC.

Theorem 3.1 [13, Theorem 3.8]. Consider an impulsive semidynamical system $(X, \pi ; M, I)$. Assume that no initial point in $(X, \pi)$ belongs to the impulsive set $M$ and that each element of $M$ satisfies the condition (TC). Then $\phi$ is continuous at $x$ if and only if $x \notin M$.

Now, consider an impulsive semidynamical system $(X, \pi ; M, I)$ and $A \subset X$. We define

$$
\tilde{\pi}^{+}(A)=\bigcup_{x \in A} \tilde{\pi}^{+}(x) \text { and } \tilde{\pi}(A, t)=\bigcup_{x \in A} \tilde{\pi}(x, t)
$$

for each $t \geq 0$. If $\widetilde{\pi}^{+}(A) \subset A$, we say that $A$ is positively $\widetilde{\pi}$-invariant. And, a set $A$ is $I$-invariant if $I(x) \in A$ for all $x \in A \cap M$.

The limit set of $A \subset X$ in $(X, \pi ; M, I)$ is given by

$$
\begin{array}{r}
\widetilde{L}^{+}(A)=\left\{y \in X: \text { there exist sequences }\left\{x_{n}\right\}_{n \geq 1} \subset A \text { and }\left\{t_{n}\right\}_{n \geq 1} \subset \mathbb{R}_{+}\right. \\
\text {such that } \left.t_{n} \stackrel{n \rightarrow+\infty}{\longrightarrow}+\infty \text { and } \widetilde{\pi}\left(x_{n}, t_{n}\right) \stackrel{n \rightarrow+\infty}{\longrightarrow} y\right\} .
\end{array}
$$

The prolongational limit set of $A \subset X$ is given by

$$
\begin{aligned}
& \widetilde{J}^{+}(A)=\left\{y \in X: \text { there are sequences }\left\{x_{n}\right\}_{n \geq 1} \subset X \text { and }\left\{t_{n}\right\}_{n \geq 1} \subset \mathbb{R}_{+}\right. \\
& \left.\quad \text { such that } d\left(x_{n}, A\right) \stackrel{n \rightarrow+\infty}{\longrightarrow} 0, t_{n} \stackrel{n \rightarrow+\infty}{\longrightarrow}+\infty \text { and } \widetilde{\pi}\left(x_{n}, t_{n}\right) \stackrel{n \rightarrow+\infty}{\longrightarrow} y\right\}
\end{aligned}
$$

and the prolongation set of $A \subset X$ is given by

$$
\begin{array}{r}
\widetilde{D}^{+}(A)=\left\{y \in X: \text { there exist sequences }\left\{x_{n}\right\}_{n \geq 1} \subset X \text { and }\left\{t_{n}\right\}_{n \geq 1} \subset \mathbb{R}_{+}\right. \\
\text {such that } \left.d\left(x_{n}, A\right) \stackrel{n \rightarrow+\infty}{\longrightarrow} 0 \text { and } \widetilde{\pi}\left(x_{n}, t_{n}\right) \stackrel{n \rightarrow+\infty}{\longrightarrow} y\right\} .
\end{array}
$$

If $A=\{x\}$ we set $\widetilde{L}^{+}(x)=\widetilde{L}^{+}(\{x\}), \widetilde{J}^{+}(x)=\widetilde{J}^{+}(\{x\})$ and $\widetilde{D}^{+}(x)=\widetilde{D}^{+}(\{x\})$.

In the sequel, we mention some auxiliary results that will be very useful later in this paper. For each $x \in X$, the motion $\widetilde{\pi}(x, t)$ is defined for every $t \geq 0$, that is, $[0,+\infty)$ denotes the maximal interval of definition of $\widetilde{\pi}_{x}$. 
Lemma 3.2 [5, Lemma 3.2]. Given an impulsive semidynamical system $(X, \pi$; $M, I)$, suppose $w \in X \backslash M$ and $\left\{z_{n}\right\}_{n \geq 1}$ is a sequence in $X$ which converges to $w$. Then, for any $t \geq 0$ there is a sequence $\left\{\varepsilon_{n}\right\}_{n \geq 1} \subset \mathbb{R}$ such that $\varepsilon_{n} \stackrel{n \rightarrow+\infty}{\longrightarrow} 0$ and $\tilde{\pi}\left(z_{n}, t+\varepsilon_{n}\right) \stackrel{n \rightarrow+\infty}{\longrightarrow} \tilde{\pi}(w, t)$.

Lemma 3.3 [10, Lemma 3.3]. Given an impulsive semidynamical system $(X, \pi ; M, I)$, suppose $w \in X \backslash M$ and $\left\{z_{n}\right\}_{n \geq 1}$ is a sequence in $X$ which converges to $w$. Then, for any $t \geq 0$ such that $t \neq \sum_{j=0}^{k} \phi\left(w_{j}^{+}\right), k=0,1, \ldots$, we have $\widetilde{\pi}\left(z_{n}, t\right) \stackrel{n \rightarrow+\infty}{\longrightarrow} \widetilde{\pi}(w, t)$.

Lemma 3.4 [10, Lemma 3.1]. Let $(X, \pi ; M, I)$ be an impulsive semidynamical system and $x \in X$. Suppose $\phi\left(x_{j}^{+}\right)<\infty$ for every $j=0,1, \ldots$ Then

$$
\overline{\widetilde{\pi}^{+}(x)}=\widetilde{\pi}^{+}(x) \cup \widetilde{L}^{+}(x) \cup\left\{x_{j}: j=1,2, \ldots\right\},
$$

where $x_{j}=\pi\left(x_{j-1}^{+}, \phi\left(x_{j-1}^{+}\right)\right), j=1,2, \ldots$, and $\tilde{\pi}^{+}(x)$ is the positive orbit of $x$ with respect to $\widetilde{\pi}$. Note that if $\phi\left(x_{j}^{+}\right)<+\infty, j=0,1, \ldots, k$ and $\phi\left(x_{k+1}^{+}\right)=+\infty$ then

$$
\overline{\widetilde{\pi}^{+}(x)}=\widetilde{\pi}^{+}(x) \cup \widetilde{L}^{+}(x) \cup\left\{x_{j}: j=1, \ldots, k+1\right\} .
$$

A point $x \in X$ is called stationary or rest point with respect to $\tilde{\pi}$, if $\widetilde{\pi}(x, t)=$ $x$ for all $t \geq 0$, it is a periodic point with respect to $\tilde{\pi}$, if $\tilde{\pi}(x, t)=x$ for some $t>0$ and $x$ is not stationary, and $x$ is an eventually periodic point with respect to $\tilde{\pi}$, if $\tilde{\pi}(x, t)$ is periodic for some $t \geq 0$. The set $\tilde{\pi}^{+}(x), x \in X$, is said to be eventually periodic if $\tilde{\pi}(x, t)$ is an eventually periodic point for all $t \geq 0$.

A point $x \in X \backslash M$ is positively Poisson $\widetilde{\pi}$-stable if $x \in \widetilde{L}^{+}(x)$, see Definition 3.2 and Theorem 3.1 in [10].

Theorem 3.5 [10, Theorem 3.3]. Let $(X, \pi ; M, I)$ be an impulsive semidynamical system and suppose $X$ is a complete metric space. Let $x \in X \backslash M$ be positively Poisson $\tilde{\pi}$-stable and not an eventually periodic point. Then the set $\widetilde{L}^{+}(x)-\widetilde{\pi}^{+}(x)$ is dense in $\widetilde{L}^{+}(x)$.

\section{The main results}

Throughout this section we shall consider an impulsive semidynamical system $(X, \pi ; M, I)$, where $(X, d)$ is a metric space. Moreover, we shall assume the following additional hypotheses:

(H1) No initial point in $(X, \pi)$ belongs to the impulsive set $M$ and each element of $M$ satisfies the condition (STC), consequently $\phi$ is continuous on $X \backslash M$ (see Theorem 3.1).

(H2) $M \cap I(M)=\emptyset$.

(H3) For each $x \in X$, the motion $\tilde{\pi}(x, t)$ is defined for every $t \geq 0$, that is, $[0,+\infty)$ denotes the maximal interval of definition of $\widetilde{\pi}_{x}$. 
4.1. Minimal sets. In [20], Kaul defines the concept of minimality for a set $A$ in an impulsive semidynamical system $(X, \pi ; M, I)$ in the following way:

$A$ is minimal in $(X, \pi ; M, I)$ if $A=\overline{\widetilde{\pi}^{+}(x)}$ for all $x \in A \backslash M$.

However, in the classical theory of continuous dynamical systems, a set $A$ in a dynamical system $(X, \pi)$ is called minimal if $A$ is nonempty, closed, invariant and it does not contain any proper subset with these properties, see Definition 3.1 (Chapter III) from [4]. As consequence, it follows that a nonempty set $A \subset X$ is minimal if and only if $\overline{\pi^{+}(x)}=A$ for every $x \in A$, see Theorem 3.2 (Chapter III) in [4].

Thus, we define the concept of minimality for impulsive semidynamical systems as presented in [4] and we show that the definition from Kaul is equivalent to our definition.

Definition 4.1. A set $A \subset X$ is minimal in $(X, \pi ; M, I)$ if the following conditions hold:

(a) $A \backslash M \neq \emptyset$;

(b) $A$ is closed;

(c) $A \backslash M$ is positively $\widetilde{\pi}$-invariant;

(d) $A$ does not contain any proper subset satisfying (a), (b) and (c).

REMARK 4.2. Given an impulsive semidynamical system $(X, \pi ; M, I)$, we suppose that there is a point $x \in X \backslash M$ such that $I(\pi(x, \phi(x)))=x$. Then $A=\pi(x,[0, \phi(x)])$ is minimal in $(X, \pi ; M, I)$.

In the sequel, we show that the definition given by Kaul, [20], is equivalent to Definition 4.1. But before that we present an auxiliary result.

Proposition 4.3. Let $(X, \pi ; M, I)$ be an impulsive semidynamical system. Then the set $\widetilde{L}^{+}(x) \backslash M$ is positively $\widetilde{\pi}$-invariant for all $x \in X$.

Proof. Suppose $\widetilde{L}^{+}(x) \backslash M$ is nonempty. Let $y \in \widetilde{L}^{+}(x) \backslash M$ and $t \geq 0$ be arbitrary. Then there is a sequence $\left\{t_{n}\right\}_{n \geq 1} \subset \mathbb{R}_{+}$with $t_{n} \stackrel{n \rightarrow+\infty}{\longrightarrow}+\infty$ such that $\tilde{\pi}\left(x, t_{n}\right) \stackrel{n \rightarrow+\infty}{\longrightarrow} y$. Since $y \notin M$ we may assume that $\left\{\tilde{\pi}\left(x, t_{n}\right)\right\}_{n \geq 1} \subset X \backslash M$. Then by Lemma 3.2 , there is a sequence $\left\{\varepsilon_{n}\right\}_{n \geq 1} \subset \mathbb{R}$ with $\varepsilon_{n} \stackrel{n \rightarrow+\infty}{\longrightarrow} 0$ such that

$$
\widetilde{\pi}\left(x, t_{n}+t+\varepsilon_{n}\right)=\widetilde{\pi}\left(\widetilde{\pi}\left(x, t_{n}\right), t+\varepsilon_{n}\right) \stackrel{n \rightarrow+\infty}{\longrightarrow} \widetilde{\pi}(y, t) .
$$

Note that $\left\{t_{n}+t+\varepsilon_{n}\right\}_{n \geq 1} \subset \mathbb{R}_{+}$with $t_{n}+t+\varepsilon_{n} \stackrel{n \rightarrow+\infty}{\longrightarrow}+\infty$. Then $\tilde{\pi}(y, t) \in$ $\widetilde{L}^{+}(x) \backslash M$ because $I(M) \cap M=\emptyset$ (see hypothesis (H2)). Since $t \geq 0$ is arbitrary the result is proved.

Theorem 4.4. A set $A \subset X$ is minimal in $(X, \pi ; M, I)$ if and only if $A=$ $\overline{\widetilde{\pi}^{+}(x)}$ for all $x \in A \backslash M$. 
Proof. First, let us suppose that $A$ is minimal. Let $x \in A \backslash M$ be arbitrary. Since $A \backslash M$ is positively $\widetilde{\pi}$-invariant, we have $\widetilde{\pi}^{+}(x) \subset A$, and since $A$ is closed it follows that

$$
\overline{\widetilde{\pi}^{+}(x)} \subset \bar{A}=A
$$

Note that $\overline{\widetilde{\pi}^{+}(x)} \backslash M \neq \emptyset, \overline{\tilde{\pi}^{+}(x)}$ is closed and $\overline{\widetilde{\pi}^{+}(x)} \backslash M$ is positively $\widetilde{\pi}$-invariant (by Proposition 4.3 and Lemma 3.4). By minimality of $A$ we have $\overline{\widetilde{\pi}^{+}(x)}=A$.

Now, let us show the sufficient condition. Let $B \subset A$ such that $B \backslash M \neq \emptyset, B$ is closed and $B \backslash M$ is positively $\tilde{\pi}$-invariant. If $b \in B \backslash M$ then $b \in A \backslash M$ and we have $A=\overline{\widetilde{\pi}^{+}(b)}$. Since $B \backslash M$ is positively $\widetilde{\pi}$-invariant, we get $B \subset A=\overline{\widetilde{\pi}^{+}(b)} \subset$ $\bar{B}=B$, and consequently $A=B$. Hence, $A$ is minimal in $(X, \pi ; M, I)$.

We have another way to characterize minimal sets under an additional hypothesis, see the next theorem.

TheOrem 4.5. Let $A \subset X$ and suppose $\widetilde{L}^{+}(x) \backslash M \neq \emptyset$ for all $x \in A$. Then $A$ is minimal in $(X, \pi, M, I)$ if and only if $A=\widetilde{L}^{+}(x)$ for all $x \in A \backslash M$.

Proof. Let us show the necessary condition. Let $x \in A \backslash M$ be arbitrary. By Theorem 4.4 we have $\overline{\widetilde{\pi}^{+}(x)}=A$. Then $\widetilde{L}^{+}(x) \subset A$. Since $\widetilde{L}^{+}(x) \backslash M \neq \emptyset$, $\widetilde{L}^{+}(x)$ is closed, $\widetilde{L}^{+}(x) \backslash M$ is positively $\widetilde{\pi}$-invariant (Proposition 4.3 ) and $A$ is minimal, we have $\widetilde{L}^{+}(x)=A$.

Now, let us show the sufficient condition. Suppose that $A$ is not minimal, then there is a proper subset $B$ of $A$ such that $B \backslash M \neq \emptyset, B$ is closed and $B \backslash M$ is positively $\widetilde{\pi}$-invariant. Given $b \in B \backslash M$, it follows that $b \in A \backslash M$. By hypothesis we have $A=\widetilde{L}^{+}(b)$. Since $B \backslash M$ is positively $\widetilde{\pi}$-invariant, we get $B \subset A=\widetilde{L}^{+}(b) \subset \bar{B}=B$. Therefore, $A=B$ and it is a contradiction. Then $A$ is minimal and the theorem follows.

By the proof of Theorem 4.5, we have the following result.

TheOREM 4.6. Let $A \subset X$ be a minimal set and $x \in A \backslash M$ be a point such that $\widetilde{L}^{+}(x) \backslash M \neq \emptyset$. Then $A=\widetilde{L}^{+}(x)$.

Let $x \in X$. The trajectory of $x$ is continuous provided that $\widetilde{\pi}^{+}(x)$ is minimal. See the next result.

Lemma 4.7. Let $(X, \pi ; M, I)$ be an impulsive semidynamical system. If $\widetilde{\pi}^{+}(x)$ is minimal then $\tilde{\pi}^{+}(x)=\pi^{+}(x)$.

Proof. We need to show that $\phi(x)=+\infty$. Suppose that $\phi(x)<+\infty$. Set $x_{1}=\pi(x, \phi(x)) \in M$. Then $x_{1} \in \overline{\widetilde{\pi}^{+}(x)}$. By minimality of $\widetilde{\pi}^{+}(x)$ we have $\tilde{\pi}^{+}(x)$ closed. Then $x_{1} \in \tilde{\pi}^{+}(x)$. But it is a contradiction because $\tilde{\pi}(x,(0,+\infty)) \cap M=\emptyset$ for all $x \in X$ as $I(M) \cap M=\emptyset$. Therefore, $\phi(x)=+\infty$. 
Given a continuous dynamical system it is known that every nonempty compact invariant set contains a compact minimal set, see Theorem 4.4 (Chapter III) in [4] for instance. In the next lines, we are going to present conditions to obtain this result for impulsive systems. For that we start by defining a special type of minimality in $(X, \pi ; M, I)$.

Definition 4.8. A set $A \subset X$ is $I$-minimal in $(X, \pi ; M, I)$ if the following conditions hold:

(a) $A \backslash M \neq \emptyset$;

(b) $A$ is closed;

(c) $A \backslash M$ is positively $\widetilde{\pi}$ - invariant;

(d) $I(A \cap M) \subset A$;

(e) $A$ does not contain any proper subset satisfying the four properties above.

Definition 4.9. The impulsive set $M$ satisfies a special tube condition, we write SSTC, if $M$ satisfies the condition STC and for any $x \in M$ and for any $\lambda$-tube $F(L,[0,2 \lambda])$ through $x$ we have the condtion: if $z \in F(L, \mu), 0 \leq \mu \leq \lambda$, then there is $w \in S=M \cap F(L,[0,2 \lambda])$ such that $\pi(w, \lambda-\mu)=z$.

Lemma 4.10 below gives sufficient conditions for a limit set to be $I$-invariant.

Lemma 4.10. Let $(X, M ; M, I)$ be an impulsive semidynamical system, $M$ satisfies SSTC and assume that there exists $\varepsilon_{0}>0$ such that $\pi\left(M,\left[0, \varepsilon_{0}\right]\right) \cap$ $I(M)=\emptyset$. Then $I\left(\widetilde{L}^{+}(x) \cap M\right) \subset \widetilde{L}^{+}(x), x \in X$.

Proof. Suppose that $\widetilde{L}^{+}(x) \cap M \neq \emptyset$ for some $x \in X$. Let $a \in \widetilde{L}^{+}(x) \cap$ $M$. Then there is a sequence $\left\{t_{n}\right\}_{n \geq 1} \subset \mathbb{R}_{+}$such that $t_{n} \stackrel{n \rightarrow+\infty}{\longrightarrow}+\infty$ and $\widetilde{\pi}\left(x, t_{n}\right) \stackrel{n \rightarrow+\infty}{\longrightarrow} a$. Since $M$ satisfies the condition STC, there exists a STC-tube $F(L,[0,2 \lambda])$ through $a$ given by a section $S \subset M$ such that $S=M \cap F(L,[0,2 \lambda])$. We may assume $\lambda<\varepsilon_{0}$. Since the tube is a neighbourhood of $a$, there is $\eta>0$ such that

$$
B(a, \eta) \subset F(L,[0,2 \lambda]) .
$$

Let $A_{1}=F(L,(\lambda, 2 \lambda]) \cap B(a, \eta)$ and $A_{2}=F(L,[0, \lambda]) \cap B(a, \eta)$.

Let $\left\{\tilde{\pi}\left(x, t_{n_{k}}\right)\right\}_{k \geq 1}$ be any subsequence of $\left\{\tilde{\pi}\left(x, t_{n}\right)\right\}_{n \geq 1}$. We claim that there is a number $\ell>0$ such that $\left\{\widetilde{\pi}\left(x, t_{n_{k}}\right)\right\}_{n_{k} \geq \ell} \subset A_{1}$. In fact, suppose the contrary. For convenience we assume that $\left\{\tilde{\pi}\left(x, t_{n_{k}}\right)\right\}_{k \geq 1} \subset A_{2}$. Let $y_{k}=\widetilde{\pi}\left(x, t_{n_{k}}\right), k=$ $1,2, \ldots$ By the properties of a tube, there is $s_{k} \in[0, \lambda]$ such that $F\left(y_{k}, s_{k}\right) \subset S$ for each $k=1,2, \ldots$, that is,

$$
\pi\left(F\left(y_{k}, s_{k}\right), s_{k}\right)=y_{k},
$$

$k=1,2, \ldots$ Let $k_{0}>0$ such that $t_{n_{k}}-s_{k}>0$ for all $k \geq k_{0}$. Since $\widetilde{\pi}(x, t) \notin$ $\bigcup_{n=1}^{+\infty} F\left(y_{n}, s_{n}\right)$ for all $t>0$, because $I(M) \cap M=\emptyset$ by hypothesis (H2), there are 
$t_{k}^{\prime} \in\left(t_{n_{k}}-s_{k}, t_{n_{k}}\right]$ and $w_{k} \in M$ such that $I\left(w_{k}\right)=\widetilde{\pi}\left(x, t_{k}^{\prime}\right)$ for $k \geq k_{0}$. Also, note that

$$
I\left(w_{k}\right)=\widetilde{\pi}\left(x, t_{k}^{\prime}\right) \in F(L,[0, \lambda]) \quad \text { for } k \geq k_{0} .
$$

Since $F(L,[0, \lambda]) \subset \pi\left(M,\left[0, \varepsilon_{0}\right]\right)$ we have $I\left(w_{k}\right) \in \pi\left(M,\left[0, \varepsilon_{0}\right]\right) \cap I(M)$ for all $k \geq k_{0}$, which contradicts the hypothesis.

Hence, we may assume $\left\{\widetilde{\pi}\left(x, t_{n}\right)\right\}_{n>1} \subset A_{1}$. Thus we have

$$
\mu_{n}=\phi\left(\widetilde{\pi}\left(x, t_{n}\right)\right) \stackrel{n \rightarrow+\infty}{\longrightarrow} 0 \text { and } I\left(\pi\left(\widetilde{\pi}\left(x, t_{n}\right), \mu_{n}\right)\right) \stackrel{n \rightarrow+\infty}{\longrightarrow} I(a),
$$

that is,

$$
\widetilde{\pi}\left(x, t_{n}+\mu_{n}\right) \stackrel{n \rightarrow+\infty}{\longrightarrow} I(a) .
$$

Therefore, $I(a) \in \widetilde{L}^{+}(x)$ and the result is proved.

The next theorem gives a characterization for $I$-minimal sets.

ThEOREM 4.11. Let $(X, M ; M, I)$ be an impulsive semidynamical system, $M$ satisfies SSTC and assume that there exists $\varepsilon_{0}>0$ such that $\pi\left(M,\left[0, \varepsilon_{0}\right]\right) \cap$ $I(M)=\emptyset$. Then a set $A \subset X$ is I-minimal if and only if $A=\overline{\widetilde{\pi}^{+}(x)}$ for all $x \in A \backslash M$.

Proof. It is enough to use Lemma 4.10 and the proof of Theorem 4.4.

Corollary 4.12. Let $(X, M ; M, I)$ be an impulsive semidynamical system, $M$ satisfies $\mathrm{SSTC}$ and assume that there exists $\varepsilon_{0}>0$ such that $\pi\left(M,\left[0, \varepsilon_{0}\right]\right) \cap$ $I(M)=\emptyset$. Then $A$ is I-minimal if and only if $A$ is minimal.

Follow below the result that gives conditions for a compact set to contain a minimal set.

Theorem 4.13. Let $(X, M ; M, I)$ be an impulsive semidynamical system, $M$ satisfies SSTC and assume that there exists $\varepsilon_{0}>0$ such that $\pi\left(M,\left[0, \varepsilon_{0}\right]\right) \cap$ $I(M)=\emptyset$. Let $F \subset X$ be a compact set such that $F \backslash M$ is a nonempty positively $\widetilde{\pi}$-invariant set and $F$ is I-invariant. Then $F$ contains a minimal set.

Proof. We are going to show that $F$ contains an $I$-minimal subset. The proof follows by Zorn's Lemma. Consider the family

$$
\begin{aligned}
& \sigma=\{B \subset F: B \backslash M \neq \emptyset, B \text { is compact, } \\
&B \backslash M \text { is positively } \widetilde{\pi} \text {-invariant and } B \text { is } I \text {-invariant }\} .
\end{aligned}
$$

Note that $\sigma \neq \emptyset$ because $F \in \sigma$.

Given $B_{1}, B_{2} \in \sigma$, we define the partial ordering relation " $\leq$ " by inclusion, that is, $B_{1} \leq B_{2}$ if and only if $B_{1} \subseteq B_{2}$. Let $\sigma^{*}=\left\{B_{\lambda}\right\}_{\lambda \in \Lambda}$ be a total ordered subset of $\sigma$ by relation $\leq$. Note that $\bigcap_{i=1}^{n} B_{\lambda_{i}} \neq \emptyset$, for any $B_{\lambda_{1}}, \ldots, B_{\lambda_{n}} \in \sigma^{*}$. Set $J=\bigcap_{\lambda \in \Lambda} B_{\lambda}$. We claim that $J \in \sigma$. In fact, note that: 
(i) $J \neq \emptyset$.

Indeed, suppose that $J=\emptyset$, then $F \subset \bigcup_{\lambda \in \Lambda} B_{\lambda}^{c}$. By compactness of $F$ there are $\lambda_{1}, \ldots, \lambda_{n} \in \Lambda$ such that $F \subset \bigcup_{i=1}^{n} B_{\lambda_{i}}^{c}$. Since $B_{\lambda_{i}} \subset F$ for all $i=1, \ldots, n$, we have $\bigcap_{i=1}^{n} B_{\lambda_{i}} \subset B_{\lambda_{i}}^{c}$ for all $i=1, \ldots, n$. Then $\bigcap_{i=1}^{n} B_{\lambda_{i}}=\emptyset$, which is a contradiction. Hence, $J \neq \emptyset$.

(ii) $J \subset F$ and $J$ is compact.

(iii) $J \backslash M \neq \emptyset$.

In fact, suppose that $J \backslash M=\emptyset$. Then $J=\bigcap_{\lambda \in \Lambda} B_{\lambda} \subset M$. Let $x \in J$, then $x \in B_{\lambda} \cap M$ for all $\lambda \in \Lambda$. Since each $B_{\lambda}$ is $I$-invariant we have $I(x) \in B_{\lambda} \backslash M$ for all $\lambda \in \Lambda$. Consequently, $I(x) \in J \backslash M$ which is a contradiction. Hence, $J \backslash M \neq \emptyset$.

(iv) $J \backslash M$ is positively $\tilde{\pi}$-invariant.

In fact, let $x \in J \backslash M=\bigcap_{\lambda \in \Lambda}\left(B_{\lambda} \backslash M\right)$. Then $x \in B_{\lambda} \backslash M$ for all $\lambda \in \Lambda$ and since $B_{\lambda} \backslash M$ is positively $\tilde{\pi}$-invariant we have

$$
\tilde{\pi}(x, t) \in B_{\lambda} \backslash M \quad \text { for all } t \geq 0 \text { and all } \lambda \in \Lambda .
$$

Thus

$$
\widetilde{\pi}(x, t) \in \bigcap_{\lambda \in \Lambda}\left(B_{\lambda} \backslash M\right)=J \backslash M \quad \text { for all } t \geq 0 .
$$

Since $x \in J \backslash M$ is arbitrary, it follows that $J \backslash M$ is positively $\tilde{\pi}$-invariant.

(v) $J$ is $I$-invariant.

Let $x \in J \cap M$, then $x \in B_{\lambda} \cap M$ for all $\lambda \in \Lambda$. Since each $B_{\lambda}$ is $I$-invariant we have $I(x) \in B_{\lambda}$ for all $\lambda \in \Lambda$, that is, $I(x) \in J$.

In conclusion, $J=\bigcap_{\lambda \in \Lambda} B_{\lambda} \in \sigma$.

On the other hand, if $B \in \sigma^{*}$ then $J \subseteq B$. Thus by Zorn's Lemma it follows that $\sigma$ admits a minimal element that is the $I$-minimal subset of $F$. By Corollary 4.12 this $I$-minimal set is a minimal set.

TheOREM 4.14. Let $(X, M ; M, I)$ be an impulsive semidynamical, $M$ satisfies condition SSTC system and assume that there exists $\varepsilon_{0}>0$ such that $\pi\left(M,\left[0, \varepsilon_{0}\right]\right) \cap I(M)=\emptyset$. Let $x \in X$ be such that $\overline{\widetilde{\pi}^{+}(x)}$ is compact and $\widetilde{L}^{+}(x) \backslash$ $M \neq \emptyset$. Then $\widetilde{L}^{+}(x)$ contains a compact minimal set.

Proof. Since $\overline{\widetilde{\pi}^{+}(x)}$ is compact it follows that $\widetilde{L}^{+}(x)$ is nonempty and compact. By hypotheses, we have $\widetilde{L}^{+}(x) \backslash M \neq \emptyset$. Since $\widetilde{L}^{+}(x) \backslash M$ is positively $\widetilde{\pi}$-invariant and $\widetilde{L}^{+}(x)$ is $I$-invariant (Lemma 4.10), it follows by Theorem 4.13 that $\widetilde{L}^{+}(x)$ contains a minimal set. 
4.2. Almost periodic and recurrent motions. In this section, we define the concepts of almost periodic and recurrent motions in impulsive semidynamical systems. Then we establish results which encompass these concepts.

Definition 4.15. A point $x \in X$ is said to be $\widetilde{\pi}$-recurrent if for every $\varepsilon>0$ there exists a $T=T(\varepsilon)>0$, such that for every $t, s \geq 0$, the interval $[0, T]$ contains a number $\tau>0$ such that

$$
d(\tilde{\pi}(x, t), \tilde{\pi}(x, s+\tau))<\varepsilon .
$$

A positive orbit $\widetilde{\pi}^{+}(x)$ is said to be $\widetilde{\pi}$-recurrent if $x \in X$ is $\widetilde{\pi}$-recurrent.

REMARK 4.16. If $x \in X$ is $\widetilde{\pi}$-recurrent then given $\varepsilon>0$ there is $T=T(\varepsilon)>0$ such that $\tilde{\pi}^{+}(x) \subset B(\tilde{\pi}(x,[t, t+T]), \varepsilon)$, for all $t \geq 0$.

The next result gives sufficient conditions for a point to be $\tilde{\pi}$-recurrent.

THEOREM 4.17. Let $(X, \pi ; M, I)$ be an impulsive semidynamical system and $A \subset X$ be a compact minimal set. If $x \in A \backslash M$, then $x$ is $\tilde{\pi}$-recurrent.

Proof. Suppose that $x$ is not $\tilde{\pi}$-recurrent. Then there are $\varepsilon>0$ and sequences $\left\{T_{n}\right\}_{n \geq 1},\left\{s_{n}\right\}_{n \geq 1},\left\{t_{n}\right\}_{n \geq 1} \subset \mathbb{R}_{+}$such that $T_{n} \stackrel{n \rightarrow+\infty}{\longrightarrow}+\infty$ and

$$
d\left(\widetilde{\pi}\left(x, t_{n}\right), \tilde{\pi}\left(x, s_{n}+\tau\right)\right) \geq \varepsilon \quad \text { for all } \tau \in\left[0, T_{n}\right], n=1,2, \ldots
$$

Since $x \in A \backslash M$ and $A \backslash M$ is positively $\tilde{\pi}$-invariant, we have

$$
\left\{\tilde{\pi}\left(x, t_{n}\right)\right\}_{n \geq 1} \subset A \backslash M \subset A \text { and }\left\{\tilde{\pi}\left(x, s_{n}+\frac{T_{n}}{2}\right)\right\}_{n \geq 1} \subset A \backslash M \subset A .
$$

By compactness of $A$, we may assume without loss of generality that

$$
\tilde{\pi}\left(x, t_{n}\right) \stackrel{n \rightarrow+\infty}{\longrightarrow} a \in A, \quad \tilde{\pi}\left(x, s_{n}+\frac{T_{n}}{2}\right) \stackrel{n \rightarrow+\infty}{\longrightarrow} b \in A .
$$

Case 1. $b \notin M$. Let $t \geq 0$ be fixed and arbitrary. First, suppose that $t \neq \sum_{j=0}^{k} \phi\left(b_{j}^{+}\right)$for all $k=0,1,2, \ldots$ In this case, by continuity of $\pi$ and $I$, there is $\delta>0$ such that if $d(y, b)<\delta$ then

$$
d(\widetilde{\pi}(y, t), \widetilde{\pi}(b, t))<\frac{\varepsilon}{3} .
$$

On the other hand, one can find a number $n_{0} \in \mathbb{N}$ such that

$$
\begin{aligned}
\frac{T_{n_{0}}}{2} & >t, \\
d\left(\widetilde{\pi}\left(x, t_{n}\right), a\right) & <\frac{\varepsilon}{3}, \quad \text { for all } n \geq n_{0}, \\
d\left(\tilde{\pi}\left(x, s_{n}+\frac{T_{n}}{2}\right), b\right) & <\delta, \quad \text { for all } n \geq n_{0} .
\end{aligned}
$$


Using (4.1), (4.2) and (4.3) we obtain

$$
\begin{aligned}
& d(\tilde{\pi}(b, t), a) \geq d\left(\tilde{\pi}\left(x, t_{n_{0}}\right), \tilde{\pi}\left(x, s_{n_{0}}+\frac{T_{n_{0}}}{2}+t\right)\right) \\
& \quad-d\left(\tilde{\pi}(b, t), \tilde{\pi}\left(\tilde{\pi}\left(x, s_{n_{0}}+\frac{T_{n_{0}}}{2}\right), t\right)\right)-d\left(\tilde{\pi}\left(x, t_{n_{0}}\right), a\right)>\varepsilon-\frac{\varepsilon}{3}-\frac{\varepsilon}{3}=\frac{\varepsilon}{3} .
\end{aligned}
$$

Since the choice of $t$ is arbitrary, we have

$$
d(\tilde{\pi}(b, t), a)>\frac{\varepsilon}{3} \text { for all } t \geq 0 \text { such that } t \neq \sum_{j=0}^{k} \phi\left(b_{j}^{+}\right), k=0,1,2, \ldots
$$

Now, suppose that there exists $k \in \mathbb{N}$ such that $t=\sum_{j=0}^{k} \phi\left(b_{j}^{+}\right)$. We can take a sequence $\left\{\lambda_{n}\right\}_{n \geq 1}$ of positive real numbers such that

$$
\lambda_{n} \stackrel{n \rightarrow+\infty}{\longrightarrow} \sum_{j=0}^{k} \phi\left(b_{j}^{+}\right) \quad \text { with } \sum_{j=0}^{k} \phi\left(b_{j}^{+}\right)<\lambda_{n}<\sum_{j=0}^{k+1} \phi\left(b_{j}^{+}\right) .
$$

Using (4.4) we have

$$
d\left(\tilde{\pi}\left(b, \lambda_{n}\right), a\right)>\frac{\varepsilon}{3} \quad \text { for } n=1,2, \ldots,
$$

SO

$$
d\left(\widetilde{\pi}\left(b, \sum_{j=0}^{k} \phi\left(b_{j}^{+}\right)\right), a\right) \geq \frac{\varepsilon}{3},
$$

since $\tilde{\pi}$ is continuous from the right.

Therefore, we can conclude that $d(\tilde{\pi}(b, t), a) \geq \varepsilon / 3$ for all $t \geq 0$, which implies that $a \notin \overline{\pi^{+}(b)}$. This is a contradiction since $A$ is minimal.

Case 2. $b \in M$. Since $M$ satisfies the condition STC, there exists a STC-tube $F(L,[0,2 \lambda])$ through $b$ given by a section $S$. Since the tube is a neighbourhood of $b$, there is $\eta>0$ such that $B(b, \eta) \subset F(L,[0,2 \lambda])$. Denote $H_{1}$ and $H_{2}$ by

$$
H_{1}=F(L,(\lambda, 2 \lambda]) \cap B(b, \eta) \quad \text { and } \quad H_{2}=F(L,[0, \lambda]) \cap B(b, \eta) .
$$

Consider $w_{n}=\tilde{\pi}\left(x, s_{n}+T_{n} / 2\right)$ and recall that $w_{n} \stackrel{n \rightarrow+\infty}{\longrightarrow} b$. Here, we need to analyse two cases: when $\left\{w_{n}\right\}_{n \geq 1}$ admits subsequence in $H_{1}$ and when $\left\{w_{n}\right\}_{n \geq 1}$ admits subsequence in $\mathrm{H}_{2}$.

(a) Suppose that the sequence $\left\{w_{n}\right\}_{n \geq 1}$ admits a subsequence $\left\{w_{n_{r}}\right\}_{r \geq 1}$ in $H_{1}$. In this case we have $\phi\left(w_{n_{r}}\right) \stackrel{r \rightarrow+\infty}{\longrightarrow} 0$. Then

$$
\tilde{\pi}\left(x, s_{n_{r}}+\frac{T_{n_{r}}}{2}+\phi\left(w_{n_{r}}\right)\right) \stackrel{r \rightarrow+\infty}{\longrightarrow} I(b) .
$$

Since $A \backslash M$ is positively $\tilde{\pi}$-invariant and $x \in A \backslash M$ we have $I(b) \in \bar{A}=A$. Consider the motion $\tilde{\pi}(I(b), t)$. Since $I(M) \cap M=\emptyset$ we have $I(b) \notin M$. By following the ideas of Case 1 we conclude that $d(\tilde{\pi}(I(b), t), a) \geq \varepsilon / 3$ for all $t \geq 0$, which implies that $a \notin \overline{\widetilde{\pi}^{+}(I(b))}$, which is a contradiction because $A$ is minimal. 
(b) Suppose now that the sequence $\left\{w_{n}\right\}_{n \geq 1}$ admits a subsequence $\left\{w_{n_{s}}\right\}_{s \geq 1}$ in $H_{2}$. Consider $0<\lambda<\phi(b)$. Since $w_{n_{s}} \stackrel{s \rightarrow+\infty}{\longrightarrow} b$, we have

$$
\tilde{\pi}\left(x, s_{n_{s}}+\frac{T_{n_{s}}}{2}+\lambda\right) \stackrel{s \rightarrow+\infty}{\longrightarrow} \tilde{\pi}(b, \lambda) .
$$

Since $x \in A \backslash M$ and $A \backslash M$ is positively $\widetilde{\pi}$-invariant, we get $b_{1}=\widetilde{\pi}(b, \lambda) \in \bar{A}=A$. Note that $b_{1} \notin M$ since $I(M) \cap M=\emptyset$. Considering the motion $\widetilde{\pi}\left(b_{1}, t\right)$, we use again the proof of Case 1 and we obtain a contradiction.

This shows that $x \in A \backslash M$ is $\tilde{\pi}$-recurrent and the theorem is proved.

Theorem 4.18 presents conditions for a point outside of the set $M$ to admit the closure of its positive orbit minimal.

THEOREM 4.18. Let $(X, \pi ; M, I)$ be an impulsive semidynamical system where $X$ is a complete metric space and $x \in X$. If $\widetilde{\pi}^{+}(x)$ is $\widetilde{\pi}$-recurrent then $\overline{\widetilde{\pi}^{+}(x)}$ is compact. Furthermore, if $x \notin M$ then $\overline{\widetilde{\pi}^{+}(x)}$ is minimal.

Proof. First, let us show that $\overline{\widetilde{\pi}^{+}(x)}$ is compact. Let $\varepsilon>0$ be given. Since $\tilde{\pi}^{+}(x)$ is $\tilde{\pi}$-recurrent there is $T=T(\varepsilon)>0$ such that

$$
d(\widetilde{\pi}(x, t), \tilde{\pi}(x,[0, T]))<\frac{\varepsilon}{2} \quad \text { for all } t \geq 0 .
$$

Let $y \in \overline{\widetilde{\pi}^{+}(x)}$, then there is a sequence $\left\{y_{n}\right\}_{n \geq 1} \subset \widetilde{\pi}^{+}(x)$ such that

$$
y_{n} \stackrel{n \rightarrow+\infty}{\longrightarrow} y \text {. }
$$

Let $y_{n}=\tilde{\pi}\left(x, t_{n}\right)$ with $t_{n} \in \mathbb{R}_{+}, n=1,2, \ldots$ Using (4.5) we have

$$
d\left(y_{n}, \tilde{\pi}(x,[0, T])\right)<\frac{\varepsilon}{2}, \quad n=1,2, \ldots
$$

When $n \rightarrow+\infty$, we get

$$
d(y, \widetilde{\pi}(x,[0, T])) \leq \frac{\varepsilon}{2} .
$$

On the other hand, since $\overline{\widetilde{\pi}(x,[0, T])}$ is compact it is totally bounded, that is, there are points $x_{1}, \ldots, x_{n} \in \overline{\widetilde{\pi}(x,[0, T])}$ such that

$$
\overline{\widetilde{\pi}(x,[0, T])} \subset \bigcup_{i=1}^{n} B\left(x_{i}, \frac{\varepsilon}{2}\right) .
$$

By compactness there is $z \in \overline{\widetilde{\pi}(x,[0, T])}$ such that

$$
d(y, z)=d(y, \overline{\widetilde{\pi}(x,[0, T])}) .
$$

By (4.8) there is $x_{i} \in \overline{\widetilde{\pi}(x,[0, T])}$ for some $i \in\{1, \ldots, n\}$ such that

$$
d\left(z, x_{i}\right)<\frac{\varepsilon}{2} \text {. }
$$

Thus using (4.7), (4.9) and (4.10) we obtain

$$
d\left(y, x_{i}\right) \leq d(y, z)+d\left(z, x_{i}\right)<\frac{\varepsilon}{2}+\frac{\varepsilon}{2}=\varepsilon .
$$


Then $\overline{\widetilde{\pi}^{+}(x)} \subset \bigcup_{i=1}^{n} B\left(x_{i}, \varepsilon\right)$, which means that $\overline{\widetilde{\pi}^{+}(x)}$ is totally bounded. Since $X$ is complete it follows that $\overline{\widetilde{\pi}^{+}(x)}$ is compact.

Now, let us show the second part of the theorem. Suppose $x \notin M$. We are going to show that $\overline{\widetilde{\pi}^{+}(x)}$ is minimal. Suppose to the contrary that there is a proper subset $A \subsetneq \overline{\widetilde{\pi}^{+}(x)}$ such that $A \backslash M \neq \emptyset, A$ is closed and $A \backslash M$ is positively $\widetilde{\pi}$-invariant.

It is clear that $x \notin A$ because $A \backslash M$ is positively $\widetilde{\pi}$-invariant and $A$ is closed. Thus $d(x, A)=d>0$. Choose $0<\varepsilon<d / 2$. Since $x$ is $\widetilde{\pi}$-recurrent there is $T=T(\varepsilon)>0$ such that for every $t, s \in \mathbb{R}_{+}$there is $\tau \in[0, T]$ with $d(\widetilde{\pi}(x, t), \widetilde{\pi}(x, s+\tau))<\varepsilon$.

Choose $q \in A \backslash M$. Since $A$ is closed and $A \backslash M$ positively $\widetilde{\pi}$-invariant, we have $\overline{\widetilde{\pi}^{+}(q)} \subset A$. Then

$$
d(x, \widetilde{\pi}(q, t)) \geq d(x, A)=d>2 \varepsilon \quad \text { for all } t \geq 0 .
$$

On the other hand, since $q \in \overline{\widetilde{\pi}^{+}(x)}$ and $q \notin M$ it follows that $q \in \widetilde{\pi}^{+}(x)$ or $q \in \widetilde{L}^{+}(x)$.

First, suppose that $q \in \widetilde{\pi}^{+}(x)$. Then $q=\widetilde{\pi}(x, s)$ for some $s>0$. By (4.11) we have $d(x, \widetilde{\pi}(x, s+t))>2 \varepsilon$ for all $t \geq 0$. It is a contradiction because $x$ is $\tilde{\pi}$-recurrent.

Second, suppose that $q \in \widetilde{L}^{+}(x)$. Then there is a sequence $\left\{\lambda_{n}\right\}_{n \geq 1} \subset \mathbb{R}_{+}$ such that $\lambda_{n} \stackrel{n \rightarrow+\infty}{\longrightarrow}+\infty$ and $\widetilde{\pi}\left(x, \lambda_{n}\right) \stackrel{n \rightarrow+\infty}{\longrightarrow} q$. By $\widetilde{\pi}$-recurrency of $x$ there is $r_{n} \in[0, T]$ such that

$$
d\left(x, \widetilde{\pi}\left(x, \lambda_{n}+r_{n}\right)\right)<\frac{\varepsilon}{2},
$$

for all $n=1,2, \ldots$ We may assume $r_{n} \stackrel{n \rightarrow+\infty}{\longrightarrow} r \in[0, T]$.

Since $r_{n} \stackrel{n \rightarrow+\infty}{\longrightarrow} r, \widetilde{\pi}\left(x, \lambda_{n}\right) \stackrel{n \rightarrow+\infty}{\longrightarrow} q$ and $q \notin M$ it follows by the proof of Lemma 3.6 (Case 1) from [7] that $\widetilde{\pi}\left(x, \lambda_{n}+r_{n}\right) \stackrel{n \rightarrow+\infty}{\longrightarrow} \widetilde{\pi}(q, r)$ if $r \neq \sum_{j=0}^{k} \phi\left(q_{j}^{+}\right)$ for all $k \in \mathbb{N}$. And, if $r=\sum_{j=0}^{k} \phi\left(q_{j}^{+}\right)$for some $k \in \mathbb{N}$, then

$$
\tilde{\pi}\left(x, \lambda_{n}+r_{n}\right) \stackrel{n \rightarrow+\infty}{\longrightarrow} q_{k+1}^{+}=\widetilde{\pi}(q, r) \quad \text { or } \quad \widetilde{\pi}\left(x, \lambda_{n}+r_{n}\right) \stackrel{n \rightarrow+\infty}{\longrightarrow} q_{k+1} .
$$

When $n$ approaches $+\infty$, it follows by (4.12) and by the above convergencies that

$$
d(x, \widetilde{\pi}(q, r)) \leq \frac{\varepsilon}{2}
$$

or

$$
d\left(x, q_{k+1}\right) \leq \frac{\varepsilon}{2} .
$$


If (4.13) occurs it follows by (4.11) that $2 \varepsilon<d(x, \widetilde{\pi}(q, r)) \leq \varepsilon / 2$, which is a contradiction.

If (4.14) occurs, we take a sequence $\left\{\lambda_{n}\right\}_{n \geq 1} \subset \mathbb{R}_{+}$such that $0<\lambda_{n}<\phi\left(q_{k}^{+}\right)$ and $\lambda_{n} \stackrel{n \rightarrow+\infty}{\longrightarrow} \phi\left(q_{k}^{+}\right)$. Then by (4.11) we have

$$
d\left(x, \widetilde{\pi}\left(q, \sum_{j=-1}^{k-1} \phi\left(q_{j}^{+}\right)+\lambda_{n}\right)\right)=d\left(x, \pi\left(q_{k}^{+}, \lambda_{n}\right)\right)>2 \varepsilon,
$$

where $\phi\left(q_{-1}^{+}\right)=0$. Then $d\left(x, q_{k+1}\right)=\lim _{n \rightarrow+\infty} d\left(x, \pi\left(q_{k}^{+}, \lambda_{n}\right)\right) \geq 2 \varepsilon$ which contradicts (4.14). Therefore, $\overline{\widetilde{\pi}^{+}(x)}$ is minimal.

The next definition concerns the concept of relatively dense sets, see Definition 3.11 (Chapter III) from [4].

Definition 4.19. A set $D \subset \mathbb{R}_{+}$is called relatively dense if there is a number $L>0$ such that

$$
D \cap(\alpha, \alpha+L) \neq \emptyset \quad \text { for all } \alpha \geq 0 .
$$

Next, we present a result which relate the concept of recurrence with relatively dense sets.

THEOREM 4.20. Let $(X, \pi ; M, I)$ be an impulsive semidynamical system and $\overline{\widetilde{\pi}^{+}(x)}$ be compact for some $x \in X \backslash M$. The positive orbit $\widetilde{\pi}^{+}(x)$ is $\widetilde{\pi}$-recurrent if and only if for each $\varepsilon>0$ the set $K_{\varepsilon}=\left\{t \in \mathbb{R}_{+}: d(x, \widetilde{\pi}(x, t))<\varepsilon\right\}$ is relatively dense.

Proof. Let us show the necessary condition. Given $\varepsilon>0$ there is $T=$ $T(\varepsilon)>0$ such that

$$
d(\widetilde{\pi}(x, t), \widetilde{\pi}(x,[\alpha, \alpha+T]))<\varepsilon
$$

for all $t \geq 0$ and for all $\alpha \geq 0$. In particular, $d(x, \widetilde{\pi}(x,[\alpha, \alpha+T]))<\varepsilon$, for all $\alpha \geq 0$. Then

$$
K_{\varepsilon} \cap[\alpha, \alpha+T] \neq \emptyset \quad \text { for all } \varepsilon>0 .
$$

Therefore, $K_{\varepsilon}$ is relatively dense and $L=T$ in this case.

Now, let us show the sufficient condition. Suppose that $K_{\varepsilon}$ is relatively dense for all $\varepsilon>0$. We are going to show that $\widetilde{\pi}^{+}(x)$ is $\widetilde{\pi}$-recurrent. Since $\overline{\tilde{\pi}^{+}(x)}$ is compact it is enough to show that $\overline{\widetilde{\pi}^{+}(x)}$ is minimal, see Theorem 4.17. Suppose that $\overline{\widetilde{\pi}^{+}(x)}$ is not minimal, then there is a proper subset $A \subsetneq \overline{\widetilde{\pi}^{+}(x)}$ such that $A \backslash M \neq \emptyset, A$ is closed and $A \backslash M$ is positively $\widetilde{\pi}$-invariant.

Note that $x \notin A$ because $A \backslash M$ is positively $\widetilde{\pi}$-invariant and $A$ is closed. Thus $d(x, A)=d>0$. Choose $0<\varepsilon<d / 2$. Then there is $T=T(\varepsilon)>0$ such that

$$
K_{\varepsilon} \cap[\alpha, \alpha+T] \neq \emptyset \quad \text { for all } \alpha \geq 0 .
$$


Take $q \in A \backslash M$. Since $A \backslash M$ is positively $\widetilde{\pi}$-invariant, we have $\widetilde{\pi}^{+}(q) \subset A$. Then $d=d(x, A) \leq d(x, \widetilde{\pi}(q, t))$, for all $t \geq 0$. Hence,

$$
d(x, \widetilde{\pi}(q, t))>2 \varepsilon \text { for all } t \geq 0 .
$$

Since $q \in \overline{\widetilde{\pi}^{+}(x)}$ and $q \notin M$, it follows that $q \in \widetilde{\pi}^{+}(x)$ or $q \in \widetilde{L}^{+}(x)$ (see Lemma 3.4).

First, suppose that $q \in \widetilde{\pi}^{+}(x)$. Then $q=\widetilde{\pi}(x, s)$ for some $s>0$. Then by (4.16) we have $d(x, \widetilde{\pi}(x, s+t))>2 \varepsilon$ for all $t \geq 0$. Thus $K_{\varepsilon} \cap[s, s+T]=\emptyset$ which contradicts (4.15).

Now suppose that $q \in \widetilde{L}^{+}(x)$. Then there is a sequence $\left\{\lambda_{n}\right\}_{n \geq 1} \subset \mathbb{R}_{+}$such that $\lambda_{n} \stackrel{n \rightarrow+\infty}{\longrightarrow}+\infty$ and $\widetilde{\pi}\left(x, \lambda_{n}\right) \stackrel{n \rightarrow+\infty}{\longrightarrow} q$. By hypothesis, for each $\lambda_{n}$, there is $\eta_{n} \in[0, T]$ such that

$$
d\left(x, \widetilde{\pi}\left(x, \lambda_{n}+\eta_{n}\right)\right)<\varepsilon,
$$

$n=1,2, \ldots$ We may assume without loss of generality that $\eta_{n} \stackrel{n \rightarrow+\infty}{\longrightarrow} \eta$. Since $\tilde{\pi}\left(x, \lambda_{n}\right) \stackrel{n \rightarrow+\infty}{\longrightarrow} q$ and $q \notin M$ it follows by the proof of Lemma 3.6 (Case 1) from [7] that $\widetilde{\pi}\left(x, \lambda_{n}+\eta_{n}\right) \stackrel{n \rightarrow+\infty}{\longrightarrow} \widetilde{\pi}(q, \eta)$ if $\eta \neq \sum_{j=0}^{k} \phi\left(q_{j}^{+}\right)$for all $k \in \mathbb{N}$. If $\eta=\sum_{j=0}^{k} \phi\left(q_{j}^{+}\right)$for some $k \in \mathbb{N}$ then

$$
\widetilde{\pi}\left(x, \lambda_{n}+\eta_{n}\right) \stackrel{n \rightarrow+\infty}{\longrightarrow} q_{k+1}^{+}=\widetilde{\pi}(q, \eta) \quad \text { or } \quad \widetilde{\pi}\left(x, \lambda_{n}+\eta_{n}\right) \stackrel{n \rightarrow+\infty}{\longrightarrow} q_{k+1} .
$$

When $n$ approaches $+\infty$ in (4.17) we get

$$
d(x, \widetilde{\pi}(q, \eta)) \leq \varepsilon
$$

or

$$
d\left(x, q_{k+1}\right) \leq \varepsilon .
$$

If (4.18) occurs it follows by (4.16) that $2 \varepsilon<d(x, \widetilde{\pi}(q, \eta)) \leq \varepsilon$, which is a contradiction.

However, if (4.19) occurs, we take a sequence $\left\{\mu_{n}\right\}_{n \geq 1} \subset \mathbb{R}_{+}$such that $0<\mu_{n}<\phi\left(q_{k}^{+}\right)$and $\mu_{n} \stackrel{n \rightarrow+\infty}{\longrightarrow} \phi\left(q_{k}^{+}\right)$. Then by (4.16) we have

$$
d\left(x, \widetilde{\pi}\left(q, \sum_{j=-1}^{k-1} \phi\left(q_{j}^{+}\right)+\mu_{n}\right)\right)=d\left(x, \pi\left(q_{k}^{+}, \mu_{n}\right)\right)>2 \varepsilon
$$

where $\phi\left(q_{-1}^{+}\right)=0$. It implies that $d\left(x, q_{k+1}\right)=\lim _{n \rightarrow+\infty} d\left(x, \pi\left(q_{k}^{+}, \mu_{n}\right)\right) \geq 2 \varepsilon$, which contradicts (4.19).

Therefore, $\overline{\widetilde{\pi}^{+}(x)}$ is minimal and by Theorem 4.17 it follows that $\widetilde{\pi}^{+}(x)$ is $\widetilde{\pi}$-recurrent. 
Definition 4.21. A point $x \in X$ is said to be almost $\tilde{\pi}$-periodic if for every $\varepsilon>0$, there exists a $T=T(\varepsilon)>0$ such that for every $\alpha \geq 0$, the interval $[\alpha, \alpha+T]$ contains a number $\tau=\tau(\alpha)>0$ such that $d(\tilde{\pi}(x, t), \tilde{\pi}(x, t+\tau))<\varepsilon$ for all $t \geq 0$.

Lemma 4.22. If $x \in X$ is almost $\tilde{\pi}$-periodic, then every point $y \in \tilde{\pi}^{+}(x)$ is also almost $\widetilde{\pi}$-periodic.

Proof. Let $x \in X$ be almost $\widetilde{\pi}$-periodic and $\varepsilon>0$ be given. Then there is $T=T(\varepsilon)>0$ such that for all $\alpha \geq 0$ the interval $[\alpha, \alpha+T]$ contains a number $\tau=\tau(\alpha)>0$ such that

$$
d(\tilde{\pi}(x, t), \tilde{\pi}(x, t+\tau))<\varepsilon \text { for all } t \geq 0 .
$$

Take $y \in \tilde{\pi}^{+}(x)$, then $y=\tilde{\pi}(x, s)$ for some $s \geq 0$. For each $\alpha \geq 0$ consider the number $\tau>0$ chosen above, then by (4.20) we have

$$
\begin{aligned}
d(\widetilde{\pi}(y, t), \widetilde{\pi}(y, t+\tau)) & =d(\widetilde{\pi}(\widetilde{\pi}(x, s), t), \widetilde{\pi}(\widetilde{\pi}(x, s), t+\tau)) \\
& =d(\widetilde{\pi}(x, s+t), \widetilde{\pi}(x, s+t+\tau))<\varepsilon,
\end{aligned}
$$

for all $t \geq 0$. Therefore, for all $\alpha \geq 0$ the interval $[\alpha, \alpha+T]$ contains a number $\tau=\tau(\alpha)>0$ such that $d(\tilde{\pi}(y, t), \tilde{\pi}(y, t+\tau))<\varepsilon$ for all $t \geq 0$, that is, $y \in \tilde{\pi}^{+}(x)$ is almost $\widetilde{\pi}$-periodic.

The next result shows sufficient conditions for an almost $\widetilde{\pi}$-periodic point to be $\tilde{\pi}$-recurrent.

THEOREM 4.23. Let $(X, \pi ; M, I)$ be an impulsive semidynamical system and $\overline{\widetilde{\pi}^{+}(x)}$ be compact for some $x \in X \backslash M$. If $x$ is almost $\tilde{\pi}$-periodic then $x$ is $\tilde{\pi}$-recurrent.

Proof. Given $\varepsilon>0$, there is $T=T(\varepsilon)>0$ such that for every $\alpha \geq 0$ the interval $[\alpha, \alpha+T]$ contains a number $\tau=\tau(\alpha)>0$ such that

$$
d(\widetilde{\pi}(x, t), \widetilde{\pi}(x, t+\tau))<\varepsilon \text { for all } t \geq 0 .
$$

Consider $K_{\varepsilon}=\left\{s \in \mathbb{R}_{+}: d(x, \tilde{\pi}(x, s))<\varepsilon\right\}$. We have

$$
K_{\varepsilon} \cap[\alpha, \alpha+T] \neq \emptyset \quad \text { for all } \alpha \geq 0 .
$$

Since $\varepsilon>0$ is arbitrary it follows by Theorem 4.20 that $x$ is $\widetilde{\pi}$-recurrent.

In Theorems 4.24 and 4.25 , given $x \in X$ we suppose that $\phi\left(x_{k}^{+}\right)<+\infty$ for all $k=0,1, \ldots$ Thus the sequence $\left\{x_{k}\right\}_{k \geq 1}$ represents all the impulsive points, $x_{k+1}=\pi\left(x_{k}^{+}, \phi\left(x_{k}^{+}\right)\right), k=0,1 \ldots$ These theorems deal with the relation between periodic and eventually periodic motions. If $\phi\left(x_{k}^{+}\right)=+\infty$ for some $k \in \mathbb{N}$ the results hold. 
THEOREM 4.24. Let $(X, \pi ; M, I)$ be an impulsive semidynamical system. Suppose that $X$ is complete and $\widetilde{L}^{+}(x) \backslash M \neq \emptyset$ for some $x \in X \backslash M$. If $\widetilde{\pi}^{+}(x) \cup$ $\left\{x_{k}\right\}_{k \geq 1}$ is minimal then $x$ is eventually periodic.

Proof. Suppose that $x \in X$ is not eventually periodic. Since $\tilde{\pi}^{+}(x) \cup$ $\left\{x_{k}\right\}_{k \geq 1}$ is minimal and $\widetilde{L}^{+}(x) \backslash M \neq \emptyset$ it follows by Theorem 4.6 that $\widetilde{\pi}^{+}(x) \cup$ $\left\{x_{k}\right\}_{k \geq 1}=\widetilde{L}^{+}(x)$. Consequently, $x \in \widetilde{L}^{+}(x)$, that is , $x$ is positively Poisson $\widetilde{\pi}$-stable. Then by Theorem 3.5 we have

$$
\overline{\widetilde{L}^{+}(x)-\widetilde{\pi}^{+}(x)}=\widetilde{L}^{+}(x) .
$$

Thus $\overline{\left\{x_{k}\right\}_{k \geq 1}}=\widetilde{L}^{+}(x)$ which is a contradiction since $\widetilde{L}^{+}(x) \backslash M \neq \emptyset$. Therefore, $x$ is eventually periodic.

THEOREM 4.25. Let $(X, \pi ; M, I)$ be an impulsive semidynamical system and $x \in X \backslash M$ be such that $x \in \widetilde{L}^{+}(x)$. If $x$ is eventually periodic then $x$ is periodic.

Proof. Since $x$ is eventually periodic there is $t \geq 0$ such that $\tilde{\pi}(x, t)$ is periodic. Thus there is $T>0$ such that $\tilde{\pi}(x, t+T)=\widetilde{\pi}(x, t)$.

Case 1. $T \neq \sum_{j=0}^{k} \phi\left(x_{j}^{+}\right)$for all $k \in \mathbb{N}$.

By hypothesis we have $x \in \widetilde{L}^{+}(x)$, then there is a sequence $\left\{t_{n}\right\}_{n \geq 1} \subset \mathbb{R}_{+}$, $t_{n} \stackrel{n \rightarrow+\infty}{\longrightarrow}+\infty$ such that

$$
\widetilde{\pi}\left(x, t_{n}\right) \stackrel{n \rightarrow+\infty}{\longrightarrow} x .
$$

Since $x \notin M$ and $T \neq \sum_{j=0}^{k} \phi\left(x_{j}^{+}\right)$for all $k \in \mathbb{N}$, it follows by Lemma 3.3 that

$$
\widetilde{\pi}\left(x, t_{n}+T\right) \stackrel{n \rightarrow+\infty}{\longrightarrow} \widetilde{\pi}(x, T) .
$$

On the other hand, there is $n_{0} \in \mathbb{N}$ such that $t_{n} \geq t$ for all $n \geq n_{0}$. Moreover, we have

$$
\widetilde{\pi}\left(x, t_{n}+T\right)=\widetilde{\pi}\left(x, t_{n}\right) \text { for all } n \geq n_{0} .
$$

When $n \rightarrow+\infty$ in the above equality and using (4.21) and (4.22) we obtain $\tilde{\pi}(x, T)=x$. Then $x$ is periodic.

Case 2. $T=\sum_{j=0}^{k} \phi\left(x_{j}^{+}\right)$for some $k \in \mathbb{N}$.

Take $\varepsilon>0$ such that $\varepsilon<\min \left\{\phi(x), \phi\left(x_{k+1}^{+}\right)\right\}$and $T+\varepsilon<\sum_{j=0}^{k+1} \phi\left(x_{j}^{+}\right)$.

Since $x \in \widetilde{L}^{+}(x)$, there is a sequence $\left\{t_{n}\right\}_{n \geq 1} \subset \mathbb{R}_{+}, t_{n} \stackrel{n \rightarrow+\infty}{\longrightarrow}+\infty$ such that $\tilde{\pi}\left(x, t_{n}\right) \stackrel{n \rightarrow+\infty}{\longrightarrow} x$. Since $x \notin M$ and $0<\varepsilon<\phi(x)$ it follows by Lemma 3.3 that

$$
\tilde{\pi}\left(x, t_{n}+\varepsilon\right) \stackrel{n \rightarrow+\infty}{\longrightarrow} \tilde{\pi}(x, \varepsilon) .
$$


Also, since $\sum_{j=0}^{k} \phi\left(x_{j}^{+}\right)<T+\varepsilon<\sum_{j=0}^{k+1} \phi\left(x_{j}^{+}\right)$it follows again by Lemma 3.3 that

$$
\tilde{\pi}\left(x, t_{n}+T+\varepsilon\right) \stackrel{n \rightarrow+\infty}{\longrightarrow} \tilde{\pi}(x, T+\varepsilon) .
$$

On the other hand, there is $n_{0} \in \mathbb{N}$ such that $t_{n} \geq t$ for all $n \geq n_{0}$. This implies that $t_{n}+\varepsilon>t$ for all $n \geq n_{0}$. Since $\tilde{\pi}(x, t)$ is periodic with period $T$ we obtain

$$
\tilde{\pi}\left(x, t_{n}+\varepsilon+T\right)=\tilde{\pi}\left(x, t_{n}+\varepsilon\right) \quad \text { for all } n \geq n_{0} .
$$

When $n \rightarrow+\infty$ in the above equality and take in account (4.23) and (4.24) we get $\tilde{\pi}(x, T+\varepsilon)=\tilde{\pi}(x, \varepsilon)$. Since $\tilde{\pi}$ is continuous from the right and $\varepsilon>0$ is arbitrary we have

$$
\tilde{\pi}(x, T)=\lim _{\varepsilon \rightarrow 0^{+}} \tilde{\pi}(x, T+\varepsilon)=\lim _{\varepsilon \rightarrow 0^{+}} \tilde{\pi}(x, \varepsilon)=x .
$$

Thus $x$ is periodic.

According to Theorems 4.24 and 4.25 , we obtain the following result.

Corollary 4.26. Let $(X, \pi ; M, I)$ be an impulsive semidynamical system. Suppose $X$ complete and let $x \in X \backslash M$ be such that $\widetilde{L}^{+}(x) \backslash M \neq \emptyset$. If $\widetilde{\pi}^{+}(x) \cup$ $\left\{x_{k}\right\}_{k \geq 1}$ is minimal then $x$ is periodic.

Acknowledgements. We thank the anonymous referee for the useful suggestions.

\section{REFERENCES}

[1] N.U. Ahmed, Existence of optimal controls for a general class of impulsive systems on Banach spaces, SIAM J. Control Optim. 42 (2003), 669-685.

[2] R. Ambrosino, F. Calabrese, C. Cosentino and G. De Tommasi, Sufficient conditions for finite-time stability of impulsive dynamical systems, IEEE Trans. Automat. Control 54 (2009), 861-865.

[3] L. Barreira And C. VAlls, Lyapunov regularity of impulsive differential equations, J. Differential Equations 249 (2010), 1596-1619.

[4] N.P. Bhatia And G.P. Szegö, Stability Theory of Dynamical Systems, Grundlehren Math. Wiss. Band 161, Springer-Verlag, New York, 1970; reprint of the 1970 original in: Classics Math., Springer-Verlag, Berlin, 2002.

[5] E.M. Bonotto, Flows of characteristic $0^{+}$in impulsive semidynamical systems, J. Math. Anal. Appl. 332 (2007), 81-96.

[6] _ LaSalle's Theorems in impulsive semidynamical systems, Nonlinear Anal. 71 (2009), 2291-2297.

[7] E.M. Bonotto and D.P. Demuner, Autonomous dissipative semidynamical systems with impulses, Topol. Methods Nonlinear Anal. 41 (2013), 1-38.

[8] E.M. Bonotto And M. Federson, Topological conjugation and asymptotic stability in impulsive semidynamical systems, J. Math. Anal. Appl. 326 (2007), 869-881.

[9] _ Limit sets and the Poincaré-Bendixson Theorem in semidynamical impulsive systems, J. Differential Equations 244 (2008), 2334-2349. 
[10] — Poisson stability for impulsive semidynamical systems, Nonlinear Anal. 71 (2009), 6148-6156.

[11] E.M. Bonotto And N.G. Grulha JR., Lyapunov stability of closed sets in impulsive semidynamical systems, Electron. J. Differential Equations 78 (2010), 1-18.

[12] B. Bouchard, N.-M. Dang and Ch.-A. Lehalle, Optimal control of trading algorithms: a general impulse control approach, SIAM J. Finan. Math. 2 (2011), 404-438.

[13] K. Ciesielski, On semicontinuity in impulsive dynamical systems, Bull. Polish Acad. Sci. Math. 52 (2004), 71-80.

[14] _ On stability in impulsive dynamical systems, Bull. Polish Acad. Sci. Math. 52 (2004), 81-91.

[15] _ On time reparametrizations and isomorphisms of impulsive dynamical systems, Ann. Polon. Math. 84 (2004), 1-25.

[16] J. CoRTÉs, Discontinuous dynamical systems: a tutorial on solutions, nonsmooth analysis, and stability, IEEE Control Syst. Mag. 28 (2008), 36-73.

[17] M.H.A. Davis, X. Guo and G. Wu, Impulse Control of multidimensional jump diffusions, SIAM J. Control Optim. 48 (2010), 5276-5293.

[18] J.A. Feroe, Existence and stability of multiple impulse olutions of a nerve equation, SIAM J. Appl. Math. 42 (1982), 235-246.

[19] S.K. Kaul, On impulsive semidynamical systems, J. Math. Anal. Appl. 150 (1990), 120128.

[20] _ On impulsive semidynamical systems II, Recursive properties, Nonlinear Anal. 16 (1991), 635-645.

[21] Stability and asymptotic stability in impulsive semidynamical systems, J. Appl. Math. Stoch. Anal. 7 (1994), 509-523.

[22] V. Rozko, A class of almost periodic motions in pulsed system, Differ. Uravn. 8 (1972), 2012-2022.

[23] _ Stability in terms of Lyapunov discontinuous dynamic systems, Differ. Uravn. 11 (1975), 1005-1012.

Manuscript received November 12, 2012

Everaldo M. Bonotto And Manuel Z. Jimenez

Instituto de Ciências Matemáticas e de Computação

Universidade de São Paulo

Campus de São Carlos

Caixa Postal 668

13560-970 São Carlos SP, BRAZIL

E-mail address: ebonotto@icmc.usp.br, manzulji@icmc.usp.br 